\title{
Pre-sleep protein in casein supplement or whole-food form has no impact on resting energy expenditure or hunger in women
}

\author{
Samantha M. Leyh ${ }^{1}$, Brandon D. Willingham ${ }^{1}$, Daniel A. Baur ${ }^{2}$, Lynn B. Panton ${ }^{1}$ \\ and Michael J. Ormsbee ${ }^{1,3 *}$ \\ ${ }^{1}$ Department of Nutrition, Food, and Exercise Sciences, Institute of Sport Sciences and Medicine, Florida State University, \\ Tallabassee, FL 32306, USA \\ ${ }^{2}$ Department of Exercise Science, Elon University, Elon, NC 27244, USA \\ ${ }^{3}$ Department of Biokinetics, Exercise and Leisure Sciences, University of KwaZulu-Natal, Durban 4000, South Africa
}

(Submitted 5 April 2018 - Final revision received 6 July 2018 - Accepted 1 August 2018)

\begin{abstract}
The purpose of this study was to determine the effect of a whole-food protein (cottage cheese, CC) consumed before sleep on next-morning resting energy expenditure (REE), RER and appetite compared with an isoenergetic/isonitrogenous casein protein (CP) supplement and placebo (PL) in active women. In a beverage-blinded, randomised, cross-over design, ten active women (age, 23.1 (sD 1.9) years; body fat, 22.0 (SD 4.6) \%) consumed pre-sleep CC (30 g of protein, $10 \mathrm{~g}$ of carbohydrate and $0 \mathrm{~g}$ of fat) or energy- and protein-matched liquid CP or PL $(0 \mathrm{~kJ})$. Participants arrived at 18.00 hours for an overnight stay in the laboratory. At 30-60 min before normal bed time ( $2 \mathrm{~h}$ post standard meal), participants consumed CC, CP or PL before measurement of REE. Upon waking (05.00-08.00 hours), REE was repeated and subjective appetite was recorded. Statistical analyses were conducted using repeated-measures ANOVA (SPSS). Significance was accepted at $P \leq 0 \cdot 05$. There were no significant differences in acute REE (CC, 7217 (sD 1368); CP, 7188 (sD 895); PL, 7075 (sD 1108) kJ/d, $P=0 \cdot 95$ ), acute RER (0.79 (sD 0.05), $P=0.56$ ), morning REE (CC, 5840 (sD 1225); CP, 5694 (sD 732); PL, 5991 (sD 903) kJ/d, $P=0.79$ ) or morning RER (0.77 (sD 0.03), $P=0.52$ ). Subjective measures of appetite were not different between groups. In active women, pre-sleep consumption of CC does not alter REE or RER more than a CP or PL beverage. These data suggest that the metabolic response from whole-food protein do not differ from the metabolic response of liquid protein.
\end{abstract}

\section{Key words: Night-time eating: Casein protein: Resting metabolic rate: Cottage cheese}

Recent studies have suggested that night-time hours (before sleep: following dinner but before bed) may be a window of opportunity to enhance recovery and improve whole-body metabolism with nutrition ${ }^{(1-8)}$. Night-time consumption of casein protein (CP) has been shown to enhance de novo muscle protein synthesis following resistance exercise, increase muscle protein synthesis, provide a more favourable whole-body protein balance overnight compared with fasting, increase next-morning resting energy expenditure (REE) and in some studies decrease hunger and increase satiety the following $\operatorname{morning}^{(1-5,7-13)}$.

This concept is relevant to healthy women as the current night-time feeding research has been conducted in elderly men, obese men and women, female runners and fit, college-aged men ${ }^{(1-5,7-13)}$. In a performance-based study, next-morning metabolism was probably increased after pre-sleep consumption of chocolate milk in female runners ${ }^{(3)}$. However, the influence of night-time feeding of $\mathrm{CP}$ on morning metabolism has yet to be investigated in the female population. A recent pilot study compared a dose of $54 \mathrm{~g}$ of $\mathrm{CP}$ ingested either in the morning (before 12.00 hours) or evening (90 min or less presleep) aiming to determine any differences in body composition and exercise performance in both trained men and women ${ }^{(14)}$. A total of twenty-six healthy men and women participated in the 8-week study and followed their normal exercise routine throughout ${ }^{(14)}$. Although this work was a pilot study, the authors concluded that the addition of $54 \mathrm{~g}$ of $\mathrm{CP}$, regardless of morning or evening ingestion, had no significant effects on body composition; however, direct measures of metabolism (REE) were not measured as part of the study ${ }^{(14)}$. Further investigation is required.

$\mathrm{CP}$ has characteristically been chosen for the night-time period as it is considered a slow-digesting protein and has consistently been shown to maintain low RER values, corresponding with fat metabolism ${ }^{(1-3,9,10)}$. With no observed hindrance on overnight fat metabolism, which has been confirmed by use of

Abbreviations: CC, cottage cheese; CP, casein protein; PL, placebo; REE, resting energy expenditure; TEF, thermic effect of food; VAS, visual analogue scale. 
microanalysis, casein continues to be a theoretically ideal presleep source of $\operatorname{protein}^{(10)}$.

Furthermore, the influence of whole-food protein consumed pre-sleep on metabolism is unknown. Processed food has been to shown to elicit differences in postprandial energy expenditure compared with whole food in healthy individuals ( $n 12$ women, $n 5 \mathrm{men})^{(15)}$. In a cross-over design, individuals consumed isoenergetic meals comprising either 'whole' or 'processed' cheese sandwiches; multi-grain bread and cheddar cheese were considered whole, whereas white bread and processed cheese products were considered processed. The meals were comparable in terms of macronutrient composition (protein: $15-20 \%$, carbohydrate: $40-50 \%$ and fat: 33-39\%). Postprandial energy expenditure was measured for 5-6 h after the meals and average energy expenditure for the whole sandwich meal was significantly larger than for the processed sandwich meal ${ }^{(15)}$. Authors concluded that the consumption of the processed sandwich decreases postprandial energy expenditure by nearly $50 \%$ compared with the isoenergetic wholesandwich meal ${ }^{(15)}$.

Considering these differences between processed and whole foods, we hypothesised that a pre-digested liquid supplement, being both structurally and chemically easier for the body to digest, may require less energy than consuming a whole food and produce differences in both acute and next-morning energy expenditure. Moreover, individuals may prefer more natural, whole-food options compared with manufactured shakes. However, we are currently unaware whether whole-food options confer similar potential benefits and aim to first address this concept within a healthy population. This information may help support future development of dietary strategies to promote weight loss in overweight individuals. It is worth considering that if pre-sleep feeding is a good method for maintaining healthy body composition, it could be preferable and easier to comply with for the average active, healthy population than the current popular dietary options.

Therefore, the present study aimed to compare a whole food (cottage cheese (CC)) with an isoenergetic, isonitrogenous liquid beverage (CP) to determine differences, if any, of meals that are identical in macronutrient composition but instead differ in their form of intake. CC was chosen because it is casein dominant, providing an ideal opportunity to compare with a liquid casein supplement (further detail of supplement composition is provided below). We hypothesised that CC would yield the greatest increases in REE both acutely and the following morning owing to its whole food form and therefore theoretically greater energy requirement for digestion compared with the CP beverage and, lastly, placebo (PL) beverage, owing to its non-energetic value. Further, we hypothesised no significant differences in RER across all treatments.

\section{Methods}

\section{Participants}

In this study, ten active (moderate to vigorous activity defined as $>4 \mathrm{~d}$ /week for at least 12 months) women (age, 23.1 (sD 1.9) years; height, 165.1 (SD 9.6) cm; weight, 62.0 (SD 9.5) kg; BMI, 22.0 (sD 1.6 ) kg/m²; body fat, 22.0 (sD 4.6)\%) participated. Participants were college or graduate students recruited from Florida State University. Participants were excluded if they were allergic to milk proteins, lactose intolerant, smokers (current or quit within 3 months), had an uncontrolled thyroid condition or metabolic disease, pregnant or currently taking medications or supplements known to affect metabolism. Participants were required to be eumenorrheic (on or off oral contraceptives). The participants were asked to refrain from taking any nutritional supplements (except for a multivitamin) throughout the duration of the study. Participants were also asked to maintain their usual exercise regimen and nutritional intake throughout the study. This study was conducted according to the guidelines laid down in the Declaration of Helsinki, and all procedures involving human subjects were approved by the Florida State University Institutional Review Board (2016.18644). Written informed consent was obtained from all participants before participation in the study.

\section{Study design}

This study had a partially blind (owing to knowledge of consuming CC), randomised, cross-over design (Fig. 1). Participants stayed overnight in the Florida State University Institute of Sports Sciences and Medicine (ISSM) on four separate occasions including one familiarisation visit and three experimental visits. On visit 1, participants reported to the laboratory at 18.00 hours for familiarisation of the protocol. Baseline anthropometrics including measurements of height (Seca Corporation), weight (digital scale; Detecto) and body composition (described below) via air-displacement plethysmography (Bod Pod; COSMED) were completed. Participants were also familiarised with the experimental protocol including measurement of REE and RER via indirect calorimetry (TrueOne 2400 metabolic cart;

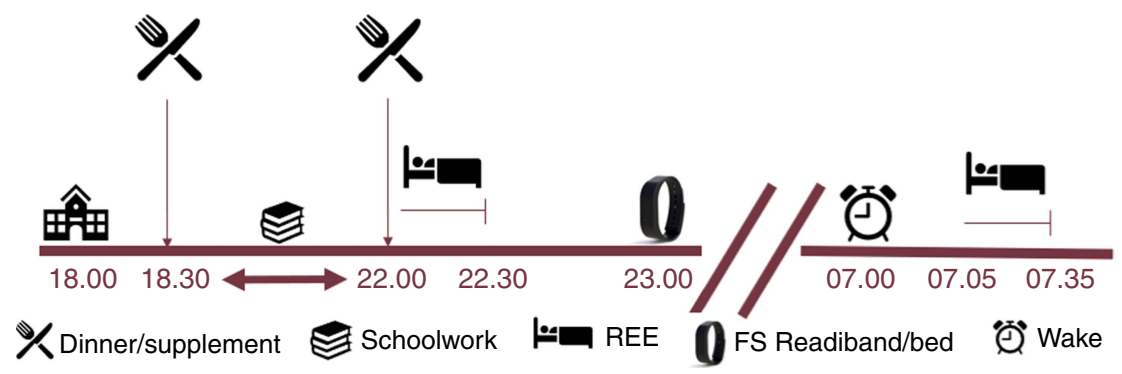

Fig. 1. Experimental design. REE, resting energy expenditure; FS, Fatigue Science; timeline is in hours. 
ParvoMedics) 30-60 min pre-sleep, staying overnight in the laboratory and next-morning subjective measurement of appetite (visual analogue scale (VAS)) and repeated metabolic measurements (REE and RER).

Visits 2-4 were for experimental testing of the three nutritional interventions (CC, CP, PL). Participants arrived at 18.00 hours for an overnight stay in the laboratory. At 18.30 hours, participants consumed a dinner meal. Participants were instructed to bring a typical dinner meal that reflected their normal dietary intake (further detail of the specific breakdown of the meals is provided below). At 30-60 min before participants' bed time and $2 \mathrm{~h}$ after their standardised meal, participants consumed CC, CP or PL before immediate (acute) measurement of REE and RER for $30 \mathrm{~min}$. After completion of experimental testing, participants went to sleep. The sleeping arrangements in the ISSM included a blow-up mattress, pillows and blankets for the participants. The same female researcher slept at the laboratory. Sleep was monitored with wrist actigraphy (Fatigue Science Inc.). Immediately upon waking the next morning (05.00-08.00 hours), following use of the restroom, subjective measures of appetite VAS were recorded and measurements of REE and RER were repeated. Visits 2-4 were separated by at least $24 \mathrm{~h}$ to ensure that participants were tested within the same menstrual cycle phase while also controlling for the maintenance of a usual physical activity regimen. All experimental visits were completed during menses to confirm that each participant was in her respective follicular phase. In all but one case, participants reported to the laboratory every other day, completing their first and last experimental session within $5 \mathrm{~d}$ (i.e. experimental session 1 on day 1 , session 2 on day 3 and session 3 on day 5). Participants were instructed to complete any physical activity by 18.00 hours the day before an experimental session, giving a full $24 \mathrm{~h}$ before arrival at the laboratory. As an example, participants arrived for day 1 at 18.00 hours and left the laboratory the following morning on day 2. They then had until 18.00 hours of day 2 to complete their physical activity. They arrived at 18.00 hours on day 3 for their second experimental session and left the next morning on day 4 to repeat the same process. For one participant, the third and final experimental visit was completed 1 month later to ensure that the participant was in the respective menstrual phase.

\section{Nutritional interventions}

The CC (Publix Supermarkets Inc.) supplement provided $669 \mathrm{~kJ}, 30 \mathrm{~g}$ of protein, $10 \mathrm{~g}$ of carbohydrate and $0 \mathrm{~g}$ of fat for the one-cup (224g) serving the participants received. CC was sent to Covance Inc. for nutrient analysis of total protein, total leucine content and sugar profile. Based on this analysis, Dymatize Nutrition Inc. produced an isoenergetic and isonitrogenous CP supplement (powder form) and a nonenergetic PL. The CP beverage therefore also provided $669 \mathrm{~kJ}, 30 \mathrm{~g}$ of protein, $10 \mathrm{~g}$ of carbohydrate and $0 \mathrm{~g}$ of fat. Both supplements (CC sand CP) were identically matched for macronutrients and micronutrients. The non-energetic PL was formulated to target the thickness, flavour and sweetness of the $\mathrm{CP}$ supplement and contained acacia gum at a level that would contribute to fewer than 10 energies post intestinal fermentation (Dymatize Nutrition Inc.). The administered dose of $30 \mathrm{~g}$ was chosen because it has previously been shown to elicit an increase in morning metabolism following pre-sleep feeding in college-aged men ${ }^{(2)}$.

\section{Anthropometrics and body composition}

Anthropometrics were assessed on the familiarisation visit. Measurements of height, weight via a digital scale and body composition via air-displacement plethysmography (BOD POD) were completed. Height and weight were measured without shoes and to the nearest $0 \cdot 1 \mathrm{~cm}$ and $0 \cdot 1 \mathrm{~kg}$, respectively. Body composition (percent body fat, lean mass and fat mass) was measured with participants wearing spandex shorts, a tightfitting top or sports bra, and a lycra cap over their hair.

\section{Dietary and exercise controls}

Participants were instructed to maintain consistent dietary and exercise habits throughout the duration of the study. Diet and exercise were controlled through self-reported data. A 2-d dietary food log and a 24-h physical activity questionnaire were completed before each visit. Furthermore, the participants were instructed to refrain from consuming caffeine or alcohol at least $24 \mathrm{~h}$ before each visit. Compliance was verified by the analysis of food logs using The Food Processor version 10.13.1 dietary software (ESHA Research) and analysis of physical activity questionnaires by the researcher. All participants consumed an identical dinner at the same time (18.30 hours) during each experimental visit. Meals were chosen by the participant to reflect a 'normal' dinner and were replicated identically for all experimental visits. Dinners chosen varied with each participant; however, they included items such as (a) chicken wrap, (b) pasta and meat sauce, and (c) sweet potato, broccoli and chicken.

\section{Sleep quality}

Participants received a Fatigue Science Readiband ${ }^{\mathrm{TM}}$ (Fatigue Science Inc.) to wear during laboratory visits. Readibands were given pre-sleep, worn for an approximately 7-10-h period and taken off before morning measurements. Participants were provided a blow-up mattress, sheets, blankets and pillows for their stay, and they were allowed to bring any further sleeping materials they desired. The room was climate-controlled, with the temperature maintained at $20-22^{\circ} \mathrm{C}$, and the same female researcher slept in the lab during all visits. The sleep variables measured included total hours slept per visit, sleep latency (length of time to transition from full wake to sleep) and sleep efficiency (percentage of time in bed sleeping).

\section{Appetite assessment}

Upon waking the following morning (05.00-08.00 hours) and after using the restroom, the participants completed a VAS to subjectively assess hunger, satiety and desire to eat. The VAS is a $100-\mathrm{mm}$ horizontal scale that consists of three lines where 
participants were asked to gauge their feeling of hunger, fullness and desire to eat, ranging from 'not at all' to 'extremely' anchored on each end of the $100-\mathrm{mm}$ line. The participants indicated their subjective feelings by marking a vertical line along the $100-\mathrm{mm}$ horizontal scale. Each rating was converted to a score in $\mathrm{mm}$ using a standardised ruler for measurement. Higher scores indicate greater feelings of each sensation.

\section{Energy expenditure and resting metabolism}

REE was measured immediately after consumption of the presleep CC, CP or PL and after completion of VAS the following morning. Participants were asked to lie supine in preparation for metabolic measurements in a dark, quiet and climatecontrolled room $\left(20-22^{\circ} \mathrm{C}\right)$. Gas exchange was collected continuously for $30 \mathrm{~min}$ to assess $\mathrm{VO}_{2}(\mathrm{ml} / \mathrm{kg}$ per $\mathrm{min}), \mathrm{REE}(\mathrm{kJ} / \mathrm{d})$ and RER via indirect calorimetry using a ventilated hood (TrueOne 2400 metabolic cart). The middle 20-min segment was used for data analysis, omitting the first 5 and last 5 min of data collection.

\section{Statistical analyses}

Sample size was estimated using G*power software (version 3.1) based on the anticipated treatment effect of protein on REE from an earlier study ${ }^{(2)}$. A power of 0.8 and an $\alpha$ level of 0.05 yielded a required sample size of 4 . Thus, our sample provided ample power. Repeated-measures ANOVA was used for the measurement of $\mathrm{VO}_{2}$, RER, hunger, satiety and desire to eat for each trial. In the case of significance, post hoc paired $t$ tests were used to assess differences between treatments. Statistical analysis was conducted using SPSS ${ }^{\circledR}$ Statistics version 21 for Windows (International Business Machines Corp.). Significance was set to $P<0.05$, and all data are reported as means and standard deviations unless otherwise specified.

\section{Results}

\section{Participants}

A total of eleven participants were screened. One participant did not meet physical activity requirements. A total of ten women from Florida State University participated in the study, and there were no dropouts. The starting date of the last menstruation was recorded for each of the participants to determine the phase of the menstrual cycle that they were in during laboratory testing using a 28-d cycle with ovulation on day 14. All participants were tested during their respective follicular phase. Descriptive characteristics of study participants are presented in Table 1.

\section{Dietary and exercise controls}

There were no differences in total energy $(P=0.75)$ or protein $(\mathrm{g} / \mathrm{kg})(P=0.84)$ consumed throughout the study. The analysis of the 48-h day dietary food logs indicated that an average of $7706(\mathrm{sD} 234) \mathrm{kJ} / \mathrm{d}$ and $1.82(\mathrm{sD} 0.06) \mathrm{g} / \mathrm{d}$ of protein was
Table 1. Characteristics of participants (Mean values and standard deviations, $n$ 10)

\begin{tabular}{lrr}
\hline & Mean & SD \\
\hline Age (years) & 23.1 & 1.9 \\
Height $(\mathrm{cm})$ & 165.1 & 3.8 \\
Weight $(\mathrm{kg})$ & 62.7 & 9.5 \\
Body fat $(\%)$ & 22.9 & 4.6 \\
BMl $\left(\mathrm{kg} / \mathrm{m}^{2}\right)$ & 22.8 & 1.6 \\
\hline
\end{tabular}

consumed over the course of the study. Average protein intake $(\mathrm{g} / \mathrm{kg})$ across treatments was as follows: CC, 1.85 (sD 0.58); CP, 1.86 (SD 0.34) and PL, 1.75 (SD 0.44). Supplementation with CC or CP significantly increased protein intake $(\mathrm{g} / \mathrm{kg})$ compared with PL: PL, 1.75 (sD 0.43); CC, 2.33 (SD 0.59) and CP, 2.34 (sD 0.36) $(P=0 \cdot 03)$. Dinner meals were specific to the individual to represent a normal dinner the participant would typically consume. The dinner meal was approximately $35 \%$ of total daily energetic intake of participants based on an average total energy intake of $7706 \mathrm{~kJ}$. Specifically, the average dinner meal was 2669 (sD 866) kJ, 55 (sD 25) g of carbohydrate, 27 (SD 13) g of fat and 45 (sD 13) g of protein. Average macronutrient composition of the dinner was $34 \%$ carbohydrate, $38 \%$ fat and $28 \%$ protein. Each participant's dinner meal before experimental testing was replicated to be exactly the same throughout the study.

\section{Sleep assessment}

Sleep in the laboratory was not different among groups. CC, CP and PL all slept for an average of 7.01 (SD 0.95) h $(P=0.44)$ and had an efficiency of 80 (sD 9$) \%(P=0.73)$.

\section{Appetite assessment}

Subjective assessments of hunger, satiety and desire to eat are presented in Fig. 2. There were no significant differences observed for hunger $(P=0 \cdot 35)$, satiety $(P=0 \cdot 26)$ or desire to eat $(P=0 \cdot 41)$ across all groups (CC, CP and PL).

\section{Energy expenditure and resting metabolism}

Acute and morning metabolic outcomes are presented in Table 2. There were no significant differences between treatments for any variables including REE, $\mathrm{VO}_{2}$ or RER in both the acute and next-morning measurements.

\section{Discussion}

This is the first study to compare consumption of a whole food $v$. a liquid supplement pre-sleep in the female population. The primary findings are that there are no differences between consuming CC (669 kJ, $30 \mathrm{~g}$ protein), CP beverage (669 kJ, $30 \mathrm{~g}$ protein) or PL beverage $(0 \mathrm{~kJ})$ on next-morning REE, RER or subjective feelings of appetite when consumed pre-sleep in healthy, physically active young women. On the basis of our findings, we reject our initial hypothesis that the whole food, CC, would cause a greater increase in metabolism. We accept 

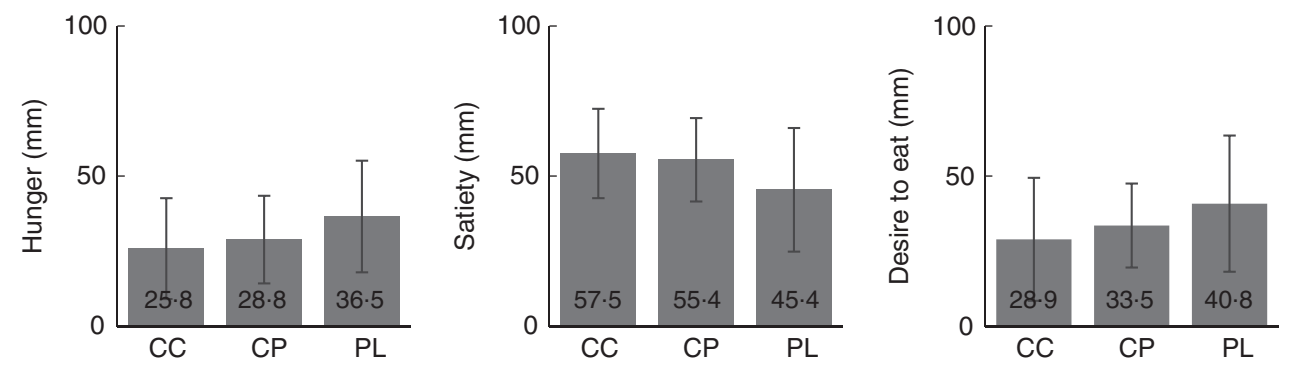

Fig. 2. Subjective appetite ratings for hunger, satiety and desire to eat the following morning. Values are means, with their standard errors represented by vertical bars. $\mathrm{CC}$, cottage cheese; $\mathrm{CP}$, casein protein; PL, placebo.

Table 2. Effects of pre-sleep cottage cheese (CC), casein protein (CP) and placebo (PL) on acute and next-morning metabolism (Mean values and standard deviations, $n 10$ )

\begin{tabular}{|c|c|c|c|c|c|c|c|}
\hline \multirow[b]{3}{*}{ Variables } & \multicolumn{7}{|c|}{ Treatment } \\
\hline & \multicolumn{2}{|c|}{$\mathrm{CC}$} & \multicolumn{2}{|c|}{$\mathrm{CP}$} & \multicolumn{2}{|c|}{ PL } & \multirow[b]{2}{*}{$P$} \\
\hline & Mean & SD & Mean & SD & Mean & SD & \\
\hline PM REE (kJ/d) & 7217 & 1368 & 7188 & 895 & 7075 & 1108 & 0.95 \\
\hline $\mathrm{PM} \mathrm{VO}_{2}(\mathrm{ml} / \mathrm{kg}$ per $\mathrm{min})$ & 3.98 & 0.40 & 4.00 & 0.46 & 3.94 & 0.50 & 0.95 \\
\hline PM RER & 0.80 & 0.06 & 0.78 & 0.04 & 0.78 & 0.06 & 0.56 \\
\hline AM REE (kJ/d) & 5840 & 1225 & 5694 & 732 & 5991 & 903 & 0.79 \\
\hline $\mathrm{AM} \mathrm{VO}_{2}(\mathrm{ml} / \mathrm{kg}$ per $\mathrm{min})$ & 3.25 & 0.42 & $3 \cdot 21$ & 0.31 & 3.35 & 0.44 & 0.70 \\
\hline AM RER & 0.78 & 0.03 & 0.78 & 0.01 & 0.76 & 0.04 & 0.52 \\
\hline
\end{tabular}

PM, pre-sleep; REE, resting energy expenditure; $A M$, upon waking.

our second hypothesis as our results have shown no difference in RER values across all treatments.

Few studies have examined the effect of pre-sleep casein supplementation in a healthy, exercising population ${ }^{(8,11,14)}$. In terms of metabolism, the results of this study are in opposition to previous research, which reported increases in next-morning REE following a protein (casein or whey) or carbohydrate, and chocolate milk supplement pre-sleep in a college-aged male population and a competitive female running population, respectively ${ }^{(2,3)}$.

Interestingly, no acute increases in $\mathrm{VO}_{2}$ were observed across any of the given treatments. Metabolic assessments in the current study were collected immediately following consumption of the interventions for a total of $30 \mathrm{~min}$. The thermic effect of food (TEF) generally peaks within $1 \mathrm{~h}$ following a meal; however, based on previous research that used a similar energy composition and the smaller energy composition of our interventions $(669 \mathrm{~kJ})$, we used the $30 \mathrm{~min}$ immediately following consumption $^{(2,16)}$. We recognise that the size and timing of the dinner meal consumed earlier (18.30 hours) may have modulated the metabolic response to the pre-sleep intervention as the thermic effect of that meal was probably still occurring. Previous research has determined that TEF of a meal lasts beyond $6 \mathrm{~h}$ for the majority of individuals ${ }^{(16)}$. Perhaps measuring for a longer period of time (i.e. $60 \mathrm{~min}$ ) may have shown different results; however, as this was a very small meal, less time would be needed. Further, our goal was to reflect a daily schedule that was realistic, choosing a typical time for the dinner meal and a normal time (chosen by the participant) for sleep. Average time getting into bed was 2230 (SD 54) min.
After pre-sleep consumption of both $\mathrm{CC}$ and $\mathrm{CP}$ beverage, each $669 \mathrm{~kJ}$, we expected increases in $\mathrm{VO}_{2}$ upon waking the following morning; however, we did not find such results. These findings are similar to night-time feeding studies using similar energy doses: $502-627 \mathrm{~kJ}$ of $\mathrm{CP}$, whey protein or carbohydrate in overweight female (age, 30.3 (sD 8.1) years) and obese male populations (age, 27.0 (SD 2.2) years) where nextmorning REE was unaffected ${ }^{(1,9,10)}$. Yet our results disagree with previous night-time feeding research in populations of college-aged active males and competitive female runners (age, 30 (SD 7) years) where $669 \mathrm{~kJ}$ of casein, whey, carbohydrate and $753 \mathrm{~kJ}$ of chocolate milk were administered, respectively, and subsequently increased next-morning $\operatorname{REE}^{(2,3)}$. We used a similar energy dose, $669 \mathrm{~kJ}$, as this previous research. Our population, although slightly younger (age, 23.1 (SD 1.9) years), was most similar to the competitive female runners who ingested chocolate milk pre-sleep and thus raises questions as to why we see differing results. These competitive female runners consumed intervention beverages that were considerably different in macronutrient make-up ${ }^{(3)}$. The interventions used in the current study comprised $669 \mathrm{~kJ}, 30 \mathrm{~g}$ of protein, $10 \mathrm{~g}$ of carbohydrate and $0 \mathrm{~g}$ of fat. The chocolate milk administered to the female runners was $753 \mathrm{~kJ}, 30 \mathrm{~g}$ of carbohydrate, $12 \mathrm{~g}$ of protein and $0 \mathrm{~g}$ of fat. There were no measurements of metabolism immediately following consumption of the chocolate milk and thus cannot be compared with our acute findings.

The administered dose of $30 \mathrm{~g}$ was chosen because it has been used in previous pre-sleep feeding studies and has shown increases in metabolism the following morning ${ }^{(2,4,11)}$. More current research has also shown that a slightly higher dose of 
$40 \mathrm{~g}$ of $\mathrm{CP}$ pre-sleep is necessary for improvements in the skeletal muscle adaptive response to resistance training in males ${ }^{(6)}$. The muscle adaptive response of this increased dose $(40 \mathrm{~g}$ ) is unknown in females. The metabolic response is also unknown and may be of significance in this active population. Of significance, it has been further concluded that a pre-sleep $30-\mathrm{g}$ dose of $\mathrm{CP}$ with $2 \mathrm{~g}$ of added free leucine was not adequate to elicit a similar skeletal muscle adaptive response as seen with the 40-g bolus in overnight muscle protein synthesis in healthy young males ${ }^{(13)}$. However, whole-body protein net balance was greater, confirming that the protein bolus is indeed being digested, absorbed and utilised by the body ${ }^{(13)}$.

In agreement with previous research, both the administered $\mathrm{CC}$ and $\mathrm{CP}$ in the present study were not different from PL in both acute and next-morning RER values, which is an indirect measurement of fuel substrate utilisation ${ }^{(2,3,9,10)}$.

Pre-sleep CP and chocolate milk (approximately $80 \%$ casein) have previously been shown to influence next-morning subjective measures of appetite, including increases in satiety and decreased desire to eat ${ }^{(1,3,9)}$. However, similar studies agree with our results, finding no significant differences between presleep treatments of $\mathrm{CP}$, whey protein, carbohydrate or PL the following morning ${ }^{(2)}$. Lack of significant differences between subjective assessments of hunger, satiety and desire to eat in the present study may be related to the high habitual dietary protein intake among the participants. Previous research has suggested that individuals with habitually high protein intakes (compared with those consuming recommended intake protein diets) may have diminished satiating response to a single protein meal $^{(17)}$. Our participants were fit, active females with dietary protein intakes well above the current RDA (1.82 (sD 0.06) g/d of protein; 25 (SD 1) \% energy (En\%) protein). Therefore, it is reasonable to assume that our participants might have blunted postprandial satiety scores compared with a similar population consuming less protein. In addition, our study may have been underpowered to assess changes in a single VAS rating of fasting appetite $^{(18)}$. Nonetheless, previous research has shown appetite changes in similar sample sizes $(n 12)^{(3,10)}$. In addition, owing to time constraints, we were unable to collect any further appetite data following the single VAS after waking. As our population consisted of undergraduate and graduate students, they had a full day of school following the sleepover session in the laboratory. However, perhaps if we were able to continue to measure appetite over multiple time points throughout the morning, we may have seen greater differences in our data between hunger, satiety and desire to eat across the interventions (CC, CP and PL).

Overall, high-protein diets have been shown to improve body composition through alterations in satiety, muscle protein synthesis and $\mathrm{TEF}^{(19)}$. The higher satiety of high-protein diets can be attributed to increases in anorexigenic hormones (cholecystokinin, glucagon-like peptide-1, peptide YY, leptin), combined with decreases in orexigenic hormones (ghrelin) ${ }^{(19)}$. Higher protein intakes have also been shown to retain lean body mass and promote fat loss in a energy-restricted state $^{(20-22)}$. Preserving lean body mass is likely to assist in body weight maintenance and REE in the long term ${ }^{(20)}$. In addition, it is well known that TEF is a smaller piece of the overall contribution to overall energy balance comprising
$3-10 \%$ of total daily energy expenditure depending on the macronutrient breakdown of the diet ${ }^{(16)}$. The main determinant of TEF is the energy content of the meal or food, followed by the protein fraction of the meal or food ${ }^{(23)}$. Protein has the highest thermic response on metabolism, with increases in metabolism ranging from 15 to $30 \%{ }^{(23)}$. High thermic effect of protein is due to necessary deamination, gluconeogenesis and urea synthesis ${ }^{(19)}$. Carbohydrates have a TEF of $5-10 \%$ and fat of $0-3 \%{ }^{(23)}$.

Therefore, through multiple mechanisms, high-protein diets effectively improve body composition ${ }^{(19)}$. Thus, determining ways to implement a high-protein diet, in this case through the addition of a pre-sleep intervention, may yield a possible strategy to aid weight loss in the overweight population. This work could aid in the future development of dietary strategies for obtaining optimal body composition. However, the alternative, as our research has shown, is that acute consumption of an additional $669 \mathrm{~kJ}$ from CP pre-sleep did not affect nextmorning appetite or REE in recreationally active females, and thus in this case would hypothetically only promote an increase in energy intake with likely no effect on energy consumption the following morning. We were unable to test this potential consequence; however, future studies should determine whether subjective measures of appetite in this population following a pre-sleep intervention would inhibit or promote further energy intake the next morning or for an entire day. On the basis of our hypothesis, this strategy and whole food could be beneficial for weight loss/maintenance, but our current data do not fully support this idea. Thus, further research is required.

\section{Limitations}

Our study design included a sleepover approach where the participants remained in the laboratory throughout the duration of each testing day, including an overnight stay until the next morning's data collection. Although this gives us a greater level of control, it may also increase sleep disturbances. We aimed to eliminate these influences through a familiarisation day. Moreover, we used Fatigue Science Readibands to measure variables of sleep quality.

\section{Conclusion}

In conclusion, these data suggest that the metabolic response from $30 \mathrm{~g}$ of whole-food protein does not differ from the metabolic response of liquid protein consumed pre-sleep in college-aged, active females. Therefore, pre-sleep feeding of protein does not cause adverse metabolic changes overnight or in next-morning metabolism.

\section{Acknowledgements}

The authors thank the participants for their dedication and participation in this study.

This study was supported by funding from the Institute of Sports Sciences and Medicine at Florida State University and support in kind supplement product from Dymatize Nutrition Inc. 
M. J. O. conceived and designed the study, secured funding for the project and oversaw data collection, data analysis and manuscript preparation. S. M. L. carried out participant recruitment, data collection and analysis and assisted with manuscript preparation. B. D. W. and D. A. B. assisted with manuscript preparation and data analysis. L. B. P. assisted with manuscript preparation and study design.

The authors have no financial or other interests concerning the outcome of the investigation. M. J. O. is on the advisory board for Clif Bar, Dymatize Nutrition and the International Protein Board. All other authors declare that they have no competing interests.

\section{References}

1. Kinsey AW, Eddy WR, Madzima TA, et al. (2014) Influence of night-time protein and carbohydrate intake on appetite and cardiometabolic risk in sedentary overweight and obese women. Br J Nutr 112, 320-327.

2. Madzima TA, Panton LB, Fretti SK, et al. (2014) Night-time consumption of protein or carbohydrate results in increased morning resting energy expenditure in active collegeaged men. Br J Nutr 111, 71-77.

3. Ormsbee MJ, Gorman KA, Miller EA, et al. (2016) Nighttime feeding likely alters morning metabolism but not exercise performance in female athletes. Appl Physiol Nutr Metab 41, 719-727.

4. Snijders T, Res PT, Smeets JSJ, et al. (2015) Protein ingestion before sleep increases muscle mass and strength gains during prolonged resistance-type exercise training in healthy young men. J Nutr 145, 1178-1184.

5. Wall BT, Burd NA, Franssen R, et al. (2016) Pre-sleep protein ingestion does not compromise the muscle protein synthetic response to protein ingested the following morning. Am J Physiol Endocrinol Metab 311, E964-E973.

6. Trommelen J \& van Loon LJC (2016) Pre-sleep protein ingestion to improve the skeletal muscle adaptive response to exercise training. Nutrients $\mathbf{8}, 763$.

7. Groen BBL, Res PT, Pennings B, et al. (2012) Intragastric protein administration stimulates overnight muscle protein synthesis in elderly men. AJP Endocrinol Metab 302, E52-E60.

8. Res PT, Groen B, Pennings B, et al. (2012) Protein ingestion before sleep improves postexercise overnight recovery. Med Sci Sports Exerc 44, 1560-1569.

9. Ormsbee MJ, Kinsey AW, Eddy WR, et al. (2015) The influence of nighttime feeding of carbohydrate or protein combined with exercise training on appetite and cardiometabolic risk in young obese women. Appl Physiol Nutr Metab 40, 37-45.

10. Kinsey AW, Cappadona SR, Panton LB, et al. (2016) The effect of casein protein prior to sleep on fat metabolism in obese men. Nutrients $\mathbf{8}, \mathrm{E} 452$.

11. Trommelen J, Holwerda AM, Kouw IWK, et al. (2016) Resistance exercise augments postprandial overnight muscle protein synthesis rates. Med Sci Sports Exerc $\mathbf{4 8}$, $2517-2525$.

12. Holwerda AM, Kouw IWK, Trommelen J, et al. (2016) Physical activity performed in the evening increases the overnight muscle protein synthetic response to presleep protein ingestion in older men. J Nutr 146, 1307-1314.

13. Trommelen J, Kouw IWK, Holwerda AM, et al. (2017) Presleep dietary protein-derived amino acids are incorporated in myofibrillar protein during post-exercise overnight recovery. Am J Physiol Endocrinol Metab 314, E457-E467.

14. Antonio J, Ellerbroek A, Peacock C, et al. (2017) Casein protein supplementation in trained men and women: morning versus evening. Int J Exerc Sci 10, 479-486.

15. Barr SB \& Wright JC (2010) Postprandial energy expenditure in whole-food and processed-food meals: Implications for daily energy expenditure. Food Nutr Res 54, 10.3402/fnr.v54i0.5144.

16. Reed GW \& Hill JO (1996) Measuring the thermic effect of food. Am J Clin Nutr 63, 164-169.

17. Long SJ, Jeffcoat AR \& Millward DJ (2000) Effect of habitual dietary-protein intake on appetite and satiety. Appetite 35, 79-88.

18. Horner KM, Byrne NM \& King NA (2014) Reproducibility of subjective appetite ratings and ad libitum test meal energy intake in overweight and obese males. Appetite 81, 116-122.

19. Morales FE, Tinsley GM \& Gordon PM (2017) Acute and longterm impact of high-protein diets on endocrine and metabolic function, body composition, and exercise-induced adaptations. J Am Coll Nutr 36, 295-305.

20. Soenen S, Martens EAP, Hochstenbach-Waelen A, et al. (2013) Normal protein intake is required for body weight loss and weight maintenance, and elevated protein intake for additional preservation of resting energy expenditure and fat free mass. J Nutr 143, 591-596.

21. Paddon-Jones D, Westman E, Mattes RD, et al. (2008) Protein, weight management, and satiety. Am J Clin Nutr 87, 1558-1561.

22. Trexler ET, Smith-Ryan AE \& Norton LE (2014) Metabolic adaptation to weight loss: implications for the athlete. J Int SOC Sports Nutr 11, 7 .

23. Westerterp KR (2004) Diet induced thermogenesis. Nutr Metab (Lond) 1, 5. 